\title{
Atitudes e embaraço face ao preservativo em estudantes de enfermagem
}

Attitudes and embarrassment about condoms in nursing students

Actitudes y vergüenza ante el preservativo en estudiantes de enfermería

Aliete Cristina Gomes Dias Pedrosa da Cunha-Oliveira' ${ }^{1}$ intps://orcid.org/0000-0001-8399-8619

Ana Paula Forte Camarneiro ${ }^{1}$ io https://orcid.org//0000-0003-3432-9261

Beatriz de Oliveira Xavier ${ }^{1}$ io https://orcid.org/0000-0002-1787-4586

Margarida Alexandra Nunes Carramanho Gomes Martins Moreira da Silva ${ }^{1}$ io https:/orcid.org/0000-0003-0031-271X

Isabel Maria Henriques Simões ${ }^{1}$ ib https://orcid.org/0000-0002-2534-9722

Ilda Maria Morais Massano Cardoso² in https://orcid.org/0000-0003-2510-2348

Como citar:

Oliveira AC, Camarneiro AP, Xavier BO, Silva MA, Simões IM, Cardoso IM. Atitudes e embaraço face ao preservativo em estudantes de enfermagem. Acta Paul Enferm. 2021;34:eAPE01954.

DOI

http://dx.doi.org/10.37689/actaape/2021A001954

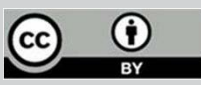

Descritores

Síndrome de imunodeficiência adquirida; Atitude; Estudantes; Preservativo; Percepção; Risco

Keywords

Acquired immunodeficiency syndrome; Attitude; Students; Condoms; Perception; Risk

Descriptores

Síndrome de inmunodeficiencia adquirida; Acttitud

Estudiantes; Condones; Percepción; Riesgo

Submetido

22 de Julho de 2019

Aceito

2 de Dezembro de 2020

Autor correspondente

Aliete Cristina Gomes Dias Pedrosa da

Cunha Oliveira

E-mail: alietecunha@esenfc.pt

\section{Resumo}

Objetivo: Conhecer a perceção do risco individual de infeção HIV; avaliar conhecimentos sobre infeção HIV; conhecer atitudes face ao uso do preservativo em função do sexo; conhecer o embaraço na aquisição, negociação e uso do preservativo em função do sexo, independentemente de preservativo masculino ou feminino.

Métodos: Estudo analítico-transversal. Amostra não probabilística constituída por 102 estudantes de Enfermagem. Instrumentos: questionário sociodemográfico e perceção do risco; teste de conhecimentos; escala de embaraço no uso do preservativo; escala de atitudes face ao uso do preservativo.

Resultados: A perceção do risco de infeção HIV é favorável/muito favorável para mais de $50 \%$ dos jovens e não estatisticamente diferente entre sexos $\left(X^{2}=2,213 ; G L=4, p=0,697\right)$. Dos jovens inquiridos, $86,3 \%$ nunca fez teste HIV e 86,1\% não se recorda de qualquer campanha de prevenção. 0 teste de conhecimentos teve resultado global de $83,7 \%$. A dimensão médico-científica mostrou os resultados mais baixos $(77,8 \%)$. 0 embaraço na aquisição do preservativo é superior nas inquiridas comparativamente com os inquiridos ( $t=$ $-2,08 ; p=0,04)$. A atitude face ao uso do preservativo não é significativamente diferente em função do sexo $(t=-1,20 ; p=0,23)$.

Conclusão: Os estudantes têm boa perceção do risco de infeção de HIV, mas baixa adesão à realização do teste e a maioria não se recorda da última campanha de prevenção. Os conhecimentos revelados são elevados, mas necessitam de ser incrementados na dimensão médico-científica. As estudantes revelam mais embaraço do que os estudantes face ao preservativo, porém, as atitudes manifestadas são idênticas. Programas formativos continuam imprescindíveis.

\section{Abstract}

Objective: To investigate the perception of individual risk of HIV infection; to assess knowledge about HIV infection; to learn about condom use attitudes according to gender; to investigate embarrassment about obtaining, negotiating and using condoms according to gender, whether male or female condom.

Method: This was an analytical cross-sectional study. A non-probabilistic sample was assembled consisting of 102 nursing students. Instruments: sociodemographic and risk perception questionnaire; knowledge test; scale of embarrassment about condom use; condom attitudes scale.

Results: Perception of risk of HIV infection is favorable/very favorable for more than $50 \%$ of young people and there was no statistical difference between the sexes $\left(X^{2}=2.213 ; G L=4, p=0.697\right)$. Of the participants, $86.3 \%$ had never been tested for HIV and $86.1 \%$ did not recall any HIV prevention campaign. The global result of the knowledge test was $83.7 \%$. The medical-scientific dimension presented the lowest results $(77.8 \%)$ 
Embarrassment about obtaining condoms was higher among the women than men ( $t=-2.08 ; p=0.04)$. Attitude towards using condoms was not significantly different between the genders ( $t=-1.20 ; p=0.23$ ).

Conclusion: The students had a good perception of the risk of HIV infection, but presented low adherence to HIV testing and most did not remember the last prevention campaign. They presented having a high level of knowledge, but they need to improve their knowledge in the medical-scientific dimension. Female students were more embarrassed than the male students about condoms, however, the attitudes towards them were identical. Educational programs continue to be essential.

\section{Resumen}

Objetivo: Conocer la percepción del riesgo individual de infección por VIH. Evaluar los conocimientos sobre infección por VIH. Conocer las actitudes ante el uso del preservativo en función del sexo. Conocer el nivel de vergüenza en la adquisición, negociación y uso de preservativos en función del sexo, tanto preservativos masculinos como femeninos.

Métodos: Estudio analítico transversal. Muestra no probabilística, formada por 102 estudiantes de enfermería. Instrumentos: cuestionario sociodemográfico y percepción del riesgo, prueba de conocimientos, escala de vergüenza en el uso de preservativos, escala de actitudes ante el uso de preservativos.

Resultados: La percepción del riesgo de infección por VIH es favorable/muy favorable para más del 50 \% de los jóvenes y no es estadísticamente diferente entre sexos $\left(X^{2}=2,213 ; G L=4, p=0,697\right)$. De los jóvenes encuestados, el 86,3 \% nunca realizó una prueba de VIH y el 86,1 \% no recuerda ninguna campaña de prevención. El resultado global de la prueba de conocimientos fue del 83,7 \%. La dimensión médico-científica tuvo los resultados más bajos (77,8 \%). La vergüenza en la adquisición de preservativos es superior en las encuestadas en comparación con los encuestados ( $t=-2,08 ; p=0,04)$. La actitud ante el uso del preservativo no es significativamente diferente en función del sexo ( $t=-1,20 ; p=0,23)$.

Conclusión: Los estudiantes tienen una buena percepción del riesgo de infección por VIH, pero una baja adherencia a la realización de pruebas y la mayoría no recuerda la última campaña de prevención. Los conocimientos revelados son altos, pero necesitan aumentar en la dimensión médico-científica. Las estudiantes revelan más vergüenza que los estudiantes ante el preservativo, pero las actitudes manifestadas son idénticas. Los programas educativos continúan siendo imprescindibles.

\section{Introdução}

As infeções sexualmente transmissíveis (IST) constituem um problema de saúde pública a nível Mundial. Segundo a Joint United Nations Program on HIV/AIDS, em 2017 cerca de 5000 pessoas foram infetadas por dia pelo HIV. Destes, 4400 eram jovens adultos com mais de 15 anos, dos quais 33\% dos $15-24$ anos e $19 \%$ do sexo feminino. ${ }^{(1)}$

Portugal apresenta das mais elevadas taxas de infeção HIV da União Europeia, com 10 casos/100 mil habitantes, comparativamente com a média de 5.7/100 mil habitantes na Uniāo Europeia, ${ }^{(2)}$ verificando-se que 33,2 \% das pessoas infetadas por HIV têm idades entre 15-29 anos, 14,6\% entre 15-24 $\operatorname{anos}^{(3)}$ e, 53,2\% são diagnósticos tardios. ${ }^{(4)}$

$\mathrm{O}$ desenvolvimento de programas educativos em saúde escolar, tem sido notável. Atualmente, cerca de $80 \%$ dos jovens têm educação sexual relacionada com esta temática, ${ }^{(5)}$ o que é de enorme importância, já que estudantes com formação têm mais probabilidades de fazer o teste HIV perante comportamentos de risco sexual. ${ }^{(5)}$ No entanto, mantêm-se áreas lacunares ao nível das atitudes, crenças erróneas individuais e coletivas e discrepância entre conhecimentos e comportamentos sexuais de risco.
Jovens do ensino superior, do curso de enfermagem, demonstram baixa perceção de risco de infeção HIV e baixa adesão ao teste HIV/AIDS. ${ }^{(6)}$ Jovens universitários em Oakland, revelaram 98\% de conhecimentos adequados sobre a transmissão do HIV, mas 53\% mostrou ideias erradas; $70 \%$ indicou o preservativo como meio de prevenção, mas apenas $28 \%$ o utilizou; $67 \%$ revelou estar sexualmente ativo, mas só $19 \%$ fez o teste HIV nos últimos dois anos, sendo a maior parte das jovens mulheres. ${ }^{(7)}$ Há evidências que estas valorizam mais o uso de preservativo do que os jovens do sexo masculino da mesma idade, ${ }^{(8)}$ Também o teste HIV é realizado com mais frequência pelo sexo feminino. ${ }^{(5)}$

Para maior eficácia na luta contra as IST, em particular o HIV/AIDS, é da máxima importância continuar a apostar na educação como meio de prevenção de comportamentos sexuais de risco, mas repensando as metodologias utilizadas. Num estudo com adolescentes, estes evidenciaram a necessidade de incluir material educacional em IST/HIV/AIDS, para ser discutido e potenciar o conhecimento, tendo indicado como prioritário discutir o uso do preservativo durante as relaçóes sexuais, de forma a colocar o foco na adesão ao mesmo. Assim, a educação tem de fazer dos educandos os seus protagonistas, de modo a construírem pensamento e sentimento de domínio sobre o tema. ${ }^{(9)}$ 
Este estudo tem como objetivos conhecer a perceção de risco individual face ao HIV em estudantes de enfermagem; avaliar o nível de conhecimentos de prevenção e transmissão da infeção HIV; conhecer as atitudes face ao uso do preservativo em função do sexo e conhecer o embaraço na aquisição, negociação e uso do preservativo em função do sexo, independentemente do tipo de preservativo, masculino ou feminino.

\section{Métodos}

Estudo quantitativo, analítico-transversal, com amostra não probabilística de conveniência, constituída por 102 estudantes do segundo ano do Curso de Graduaçáo em Enfermagem de uma escola da região Centro de Portugal, que voluntariamente aceitaram participar, após explicação dos objetivos do estudo. Constituíram critérios de inclusão, idade superior a 18 anos e de exclusão o preenchimento irregular do questionário. Recolha de dados efetuada em junho de 2019.

A colheita de dados foi realizada com o instrumento a seguir descrito. Questionário sociodemográfico e de saúde (estado serológico e perceção de risco de infeção HIV), construído para o estudo. Subteste de conhecimentos sobre prevenção de HIV/ AIDS, do Teste de Conhecimentos de Prevenção e Transmissão da Infeção VIH, de Koopman e Reid traduzido e adaptado para português ${ }^{(10)}$, constituído por 45 questóes, de resposta dicotómica, verdadeira ou falsa, que avalia três dimensóes, conhecimentos médico-científicos, mitos sobre a transmissão do HIV e conhecimentos sobre comportamentos de alto risco e, comportamentos preventivos; tem pontuação final entre zero e 100\%. Subescala de Atitudes face ao Preservativo, uma das cinco subescalas da Sexual Risks Scale, de DeHart e Birkimer traduzida e adaptada para português; ${ }^{(10)}$ contém 13 itens pontuados numa escala tipo Likert, com cinco pontos; as pontuaçôes mais altas indicam pré-disposição mais favorável a práticas de sexo seguro. Escala de Embaraço face ao Uso de Preservativo, de VailSmith e Durham, traduzida e adaptada para português, ${ }^{(11)}$ possui três dimensóes, a primeira direcionada à aquisição, compra/aquisição ou posse de pre- servativos, a segunda à negociação para o uso com o parceiro e a terceira sobre o uso propriamente dito do preservativo; é constituída por 18 itens, avaliados numa escala de concordância, tipo Likert com cinco alternativas. A pontuação varia entre 18 (nível mais baixo de embaraço) e 90 (o mais elevado).

Os dados foram processados em IBM, Statistical Pakage for Social Sciences, versão 24. Foram utilizados os testes t de Student para amostras independentes e Qui quadrado.

A pesquisa foi aprovada pelo Comissão de Ética da Unidade Investigação em Ciências da Saúde: Enfermagem, da Escola Superior de Enfermagem de Coimbra (Parecer no 596/05-2019). Os participantes assinaram o consentimento informado.

\section{Resultados}

Participaram 102 indivíduos que frequentavam o segundo ano do curso de enfermagem, de uma escola superior de Enfermagem da regiáo Centro de Portugal, com média de idades de 20,19 anos (DP= $3,81)$, dos quais $20(19,6 \%)$ eram do sexo masculino e $82(80,4 \%)$ do sexo feminino.

A perceção do risco de infeção HIV é favorável e muito favorável para mais de $50 \%$ dos jovens e não é estatisticamente diferente entre sexo $\left(X^{2}=2,213\right.$; $\mathrm{GL}=4, \mathrm{p}=0,697)$.

Dos participantes $86,3 \%$ nunca fez o teste de $\mathrm{VIH}$ e apenas $5,1 \%$ o fez nos últimos 12 meses. A perceção do risco pessoal para contrair infeção foi mais baixa no sexo masculino $(n=7 ; 35 \%)$, comparativamente com o sexo feminino $(\mathrm{n}=36 ; 44,4 \%)$.

Dos inquiridos, 83,2\% afirmou já ter participado em programas de educação para prevenção do HIV, durante o ensino secundário; $89,2 \%$ relatou ter sido "consumidor" de mensagens divulgadas pelos midia, enquanto 63,4\% recorda-se de campanhas de prevenção difundidas por outdoors ou outras formas de divulgação publicitária. Quando questionados sobre as últimas campanhas de mensagens preventivas associadas ao $\mathrm{HIV}, 86,1 \%$ não se recorda de nenhuma.

O teste de conhecimentos sobre o HIV (Tabela 1) teve, no seu global um resultado de $88,7 \%$, na 
Tabela 1. Percentagem de respostas corretas aos itens no subteste de conhecimentos sobre prevenção de HIV/AIDS

\begin{tabular}{|c|c|}
\hline Dimensões (Média global=83,7\%) & $\begin{array}{c}\text { Respostas } \\
\text { corretas em \% }\end{array}$ \\
\hline Conhecimentos médico-científicos & $\mathrm{m}=77,8$ \\
\hline 1. SIDA significa Síndrome de Imunodeficiência Adquirida & 99,0 \\
\hline 2. Hoje, a maioria dos cientistas acredita que a Sida é causada por um vírus chamado Vírus da Imunodeficiência Humana & 88,2 \\
\hline 3. A maioria das pessoas que têm Sida costumam curar-se & 97,1 \\
\hline 4. Um bebé de mãe portadora de VIH pode contrair a Sida & 87,3 \\
\hline 5. $\mathrm{OVIH}$ circula no sangue & 96,1 \\
\hline 6. A maioria das pessoas portadoras de VIH estão doentes com Sida & 62,7 \\
\hline 8. 0 VIH aparece no sémen & 93,1 \\
\hline 9. 0 número de homens e mulheres infetados com VIH será provavelmente menor nos próximos anos do que hoje & 33,3 \\
\hline 10. A Sida diminui a capacidade de resistência do organismo às doenças & 99,0 \\
\hline 12. Um teste negativo para os anticorpos VIH significa que provavelmente a pessoa tem Sida & 93,1 \\
\hline 15. Se um teste de VIH for negativo significa que a pessoa tem Sida & 96,1 \\
\hline 16. As pessoas com Sida podem, por isso, contrair outras doenças & 94,1 \\
\hline 17. Podes morrer de Sida se contraíres a doença & 36,3 \\
\hline 20. As pessoas com Sida contraem pneumonias com mais facilidade do que a média das pessoas & 93,1 \\
\hline 26. Os preservativos de pele de carneiro são melhores do que os preservativos de látex para a prevenção do VIH & 94,1 \\
\hline 31. Basta um teste VIH positivo para teres a certeza de que estás infetado(a) & 46,1 \\
\hline 33. Foi recentemente desenvolvida uma vacina que imuniza as pessoas contra o VIH & 91,2 \\
\hline 34. Mesmo com aspeto de pessoa saudável, um(a) parceiro(a) infetado(a) pode transmitir o VIH & 97,1 \\
\hline 35. Se és realmente saudável, o exercício físico poderá proteger-te da infeção pelo VIH & 98,0 \\
\hline 36. Se a pessoa com quem estiveres a fazer sexo já fez o teste do VIH e este foi negativo, isso quererá dizer que tu não estás infetado(a) & 85,3 \\
\hline 38. Melhor do que usar apenas o preservativo será usá-lo com um espermicida & 67,6 \\
\hline 39. Pode-se ser infetado pelo VIH, e depois contrair a Sida através de um golpe ou de uma ferida aberta & 37,3 \\
\hline 41. Se tiveres um teste falso positivo para o VIH, isso significa que estás infetado & 81,4 \\
\hline Mitos sobre a transmissão & $\mathrm{m}=82$ \\
\hline 11. Há pessoas que foram infetadas pelo VIH e contraíram a Sida ao terem-se sentado em sanitas públicas & 85,3 \\
\hline 23. Há pessoas que contraíram a infeção VIH por terem nadado numa piscina utilizada por alguém que tinha Sida & 96,1 \\
\hline 25. Há pessoas que foram infetadas pelo $\mathrm{VHH}$ e contraíram a Sida por terem dado beijos a uma pessoa que estava infetada & 74,5 \\
\hline 27. Pouco tempo depois de ser infetado pelo VIH, é habitual as pessoas ficarem muito doentes com Sida & 80,4 \\
\hline 29. Há pessoas que foram infetadas pelo VIH e contraíram a Sida através de picadas de insetos & 84,3 \\
\hline 32. As mulheres grávidas estão protegidas contra o VIH & 98,0 \\
\hline 37. Há pessoas que foram infetados pelo VIH por terem ido comer a um restaurante onde um dos empregados era portador de Sida. & 97,1 \\
\hline 43. Pode-se ser infetado pelo VIH, e depois contrair a Sida, ao dar sangue & 25,5 \\
\hline 45. Pode-se ser infetado pelo VIH ao fazer o teste de VIH & 97,0 \\
\hline Conhecimento sobre comportamentos de alto risco e comportamentos preventivos & $m=91,3$ \\
\hline 7. As trabalhadoras de sexo têm uma baixa probabilidade de ser infetadas pelo VIH & 98,0 \\
\hline 13. Se tiveres relações sexuais com uma só pessoa, em toda a vida, podes, ainda assim, contrair o VIH & 96,1 \\
\hline 14. Ainda que te possa mentir, é sempre boa ideia perguntares à pessoa com quem vais ter relações sexuais qual o seu passado sexual & 100 \\
\hline 18. Os homens têm mais probabilidade de contrair a infeção VIH se tiverem relações sexuais com mulheres do que se tiverem relações sexuais com homens & 99,0 \\
\hline 19. A utilização do preservativo faz diminuir a probabilidade de ser infetado pelo VIH & 98,0 \\
\hline 21. As mulheres têm mais probabilidade de ser infetadas pelo VIH se tiverem relações sexuais com um homem estritamente heterossexual do que com um homem bissexual & 93,1 \\
\hline 22. É seguro ter relações sexuais sem preservativo com uma pessoa que consome drogas injetáveis, desde que tu não te injetes & 100,0 \\
\hline 24. Qualquer pessoa, independentemente da sua raça, pode ser infetado pelo VIH & 98,0 \\
\hline 28. Contrair ou não a infeção VIH depende mais de se fazer ou não sexo seguro do que do tipo de grupo a que se pertence & 86,3 \\
\hline 30. É mais seguro não fazer sexo do que ter relações sexuais usando preservativo & 54,9 \\
\hline 40. Não há perigo de ser infetado pelo VIH e contrair a Sida se fizeres sexo oral sem preservativo & 93,1 \\
\hline 44. As pessoas que consomem álcool e drogas como a marijuana, a cocaína e o "crack" têm mais tendência para não praticar sexo seguro & 79,4 \\
\hline
\end{tabular}

dimensão médico-científica apresentou resultados mais baixos $(77,8 \%)$, na dimensão mitos sobre a transmissão, a média foi $82 \%$ e no conhecimento sobre comportamentos de alto risco e comportamentos preventivos a média foi $91,3 \%$.

Os resultados obtidos pela aplicação da Subescala de Atitudes Face ao Uso do Preservativo evidenciaram que os estudantes do sexo feminino obtiveram melhores resultados médios $(M=49,94$; $\mathrm{DP}=7,63$ ), quanto comparados com o sexo masculino $(M=47,50$; $D P=9,83)$, no entanto, esta diferença não é estatisticamente significativa $(t=-1,20$; $\mathrm{p}=0,23)$. Os resultados da Escala de Embaraço Face ao Preservativo, na sua dimensão total, mostraram valores superiores nos estudantes do sexo feminino $(\mathrm{M}=38,96 ; \mathrm{DP}=10,61)$ comparativamente aos do 
sexo masculino $(M=35,63 ; \mathrm{DP}=12,72)$, no entanto sem significado estatístico $(\mathrm{t}=-1,17 ; \mathrm{p}=0,24)$. Ainda nesta escala, as diferenças entre os sexos são significativas para a subescala de embaraço na aquisição do preservativo $(\mathrm{t}=-2,08 ; \mathrm{p}=0,04)$, sugerindo que são as jovens quem sente significativamente mais embaraço, nesta aquisição (Tabela 2).

Tabela 2. Teste $t$ de Student e U de Mann Whitney de diferenças de médias nos resultados da Escala de Embaraço Face ao uso do Preservativo em função do sexo

\begin{tabular}{|c|c|c|c|c|}
\hline Escala e subescalas & $\mathrm{m}$ & DP & $t$ & $p$-value \\
\hline \multicolumn{5}{|l|}{ Embaraço total } \\
\hline Masculino $(n=19)$ & 35,63 & 12,72 & $-1,17$ & 0,24 \\
\hline Feminino $(n=75)$ & 38,96 & 10,61 & & \\
\hline \multicolumn{5}{|l|}{ Embaraço Aquisição } \\
\hline Masculino $(n=19)$ & 18,21 & 8,60 & $-2,08$ & 0,04 \\
\hline Feminino $(n=76)$ & 22,53 & 7,92 & & \\
\hline \multicolumn{5}{|l|}{ Embaraço Negociação } \\
\hline Masculino $(n=19)$ & 7,58 & 2,87 & $-1,20$ & 0,23 \\
\hline Feminino $(n=77)$ & 8,56 & 3,25 & & \\
\hline Embaraço Uso & & & U & $p$-value \\
\hline Masculino $(n=19)$ & 9,84 & 4,27 & 541,50 & 0,06 \\
\hline Feminino $(\mathrm{n}=78)$ & 7,74 & 2,91 & & \\
\hline
\end{tabular}

\section{Discussão}

A presente investigação permitiu estudar questóes relacionadas com o conhecimento sobre HIV, perceção do risco individual do HIV, atitudes face ao uso do preservativo e embaraço face à aquisição, negociação e uso do preservativo em jovens do curso de enfermagem. Participaram um maior número de jovens do sexo feminino do que do sexo masculino, como habitualmente neste tipo de graduação.

A formação em enfermagem constitui-se um amplo campo de saberes e conhecimentos que interagem com vários domínios científicos. Apesar de estes estudantes dominarem um considerável leque de conhecimentos científicos relativamente à saúde, não significa que os comportamentos e conhecimentos sobre o HIV sejam inequivocamente corretos. Neste estudo, cerca de um quarto dos estudantes afirma que "Pode-se ser infetado pelo HIV, e depois contrair a Sida, ao dar sangue". Este dado é revelador de crenças e conhecimentos contrários ao conhecimento cientifico, revelando a presença de ideias erróneas, acarretando medos infundados. $\mathrm{O}$ mesmo acontece com os conhecimentos relacionados com o uso correto do preservativo. Sabe-se que a utilização do preservativo, quando usado de maneira correta e consistente, é eficaz na prevenção do HIV. ${ }^{(12)}$ Para tal, priorizar intervençóes dirigidas às populaçóes, promovendo o acesso ao preservativo, carece de investimento e avaliação do conhecimento relativamente ao tema. Quando questionados sobre o facto de se poder "morrer de Sida se contrair a doença”, mais de dois terços considera que não. As mortes não são diretamente resultantes da Sida em si mesma, mas das doenças ou condiçóes que resultam da imunossupressão, as quais náo estariam presentes sem a doença de base. ${ }^{(13)}$

A importância sobre o conhecimento do estado serológico das populações é fundamental. A ONUSIDA $^{(14)}$ prevê que até $2020,90 \%$ das pessoas que vivem com infeção saibam que estão infetadas, 90\% das pessoas que sabem que estáo infetadas estejam em tratamento e $90 \%$ das pessoas que estejam em tratamento tenham a infeção controlada. Com estas medidas, e de acordo com as Naçôes Unidas, prevê-se que em 2030 o HIV/AIDS seja erradicado. ${ }^{(15)}$ No entanto, a motivação da população para as medidas preventivas e conhecimentos do seu estado serológico, não parece seguir as recomendaçóes internacionais.

No presente estudo, $86,3 \%$ nunca fez o teste HIV, e apenas 5,1\% o fez nos últimos 12 meses. Estes baixos resultados são corroborados com outros estudos. ${ }^{(16)}$ Adicionalmente, a baixa perceção do risco individual para contrair infeção por HIV, variou entre os $35 \%$ para o sexo masculino e $44,4 \%$ para o sexo feminino, revelando-se um fraco preditor de prevenção e, consequentemente, uma eventual explicação para que estes estudantes não tenham realizado testes de deteção, resultados também encontrados noutras investigaçóes. ${ }^{(16)}$

O envolvimento em programas de prevenção é um indicador positivo de interesse sobre o conhecimento do HIV e os dados sugerem uma boa adesão, verificando-se que $83,2 \%$ referiu ter participado em programas de educação para a prevenção. Contudo, estes valores não implicam, necessariamente, que tenha havido mudança efetiva de comportamentos. Uma grande maioria relatou ter sido "consumidor" 
de mensagens divulgadas pelos mídia, mas não se lembra das últimas campanhas preventivas associadas ao HIV, privilegiando a recordação de campanhas difundidas através de outdoors. Em Portugal, desde 2009, está prevista na lei a natureza obrigatória de educação sexual no ensino básico e secundário. ${ }^{(17)}$ Para além disso, são seguidas as orientações da ONUSIDA, realizando-se campanhas anuais para assinalar o dia de luta contra a sida e, intensificam-se por todo o país, nas festas académicas, açôes de sensibilização e prevenção.

Os resultados sugerem que o conhecimento dos alunos foi na sua maioria elevado, no entanto, identificaram-se respostas que traduzem crenças erradas e demonstram falta de conhecimento, o que se torna preocupante quando se trata de futuros profissionais de saúde. De acordo com a literatura, ideias erróneas associadas ao uso do preservativo, bem como desconhecimento sobre a transmissão, potenciam comportamentos de risco principalmente nas mulheres. ${ }^{(18)}$

A Escala de Atitudes Face ao Uso do Preservativo revelou valores superiores no sexo feminino, comparativamente ao sexo masculino, mas sem significado estatístico. De facto, os estudantes universitários portugueses têm atitudes razoavelmente positivas face ao uso do preservativo masculino. ${ }^{(18)}$ Os resultados da presente pesquisa indiciam a necessidade de aprofundamento através de um estudo longitudinal com uma amostra mais significativa. A investigação sobre a temática tem sido amplamente debatida, apontando para que programas de prevenção devam focar-se na mudança de atitudes no sexo masculino. Esta ideia é suportada pelo facto de o uso do preservativo masculino estar, em primeira instância, associado ao sexo masculino. No entanto, as recomendaçóes evocam a necessidade de desenvolvimento de estratégias de negociação e promoção do uso do preservativo em funçáo do sexo, de forma a potenciar a aceitabilidade do seu uso como comportamento preventivo na relação com o parceiro. ${ }^{(19)}$

No que respeita ao embaraço na aquisição, negociação e uso, sabe-se que atitudes mais favoráveis determinam menor embaraço na aquisição. ${ }^{(20)}$
Nesta investigação, as estudantes demonstraram significativamente maior embaraço na aquisição do preservativo do que os estudantes, portanto, tomam menos iniciativa nessa aquisição. Também, na Escala de Embaraço, total, apresenta valores superiores, embora sem significância estatística. Foram limitaçóes ao estudo, o tamanho da amostra e a baixa representatividade do sexo masculino.

\section{Conclusão}

A maioria dos estudantes tem boa perceção do risco de infeção de HIV, não sendo diferente consoante o sexo. A realização de testes serológicos de HIV não é ainda uma realidade entre os estudantes. $\mathrm{O}$ conhecimento sobre a infeção de HIV é aceitável ou mesmo, tendencialmente, bom. Contudo, existem falsos conceitos quanto à forma de transmissão e prevenção. A atitude face ao uso do preservativo demostrou ser aceitável. Já os resultados relativos ao embaraço face à aquisição, negociação e uso do preservativo sugerem mais insegurança no sexo feminino do que no sexo masculino. Assim, propóe-se a implementação de programas formativos orientados para a mudança de comportamentos para além da aquisição de conhecimentos.

\section{Agradecimentos}

Este trabalho é financiado por Fundos Nacionais através da FCT - Fundação para a o Ciencia e Tecnologia, I.P., no âmbito do projeto Refa ${ }^{\text {.UIDB/ }}$ $00742 / 2020$.

\section{Colaborações}

Oliveira ACGDPC, Camarneiro APF, Xavier BO, Silva MANCGMM, Simóes IMH e Cardoso IMMM colaboraram com a concepção do estudo, análise e interepretação dos dados, redação do artigo, revisão crítica relevante do conteúdo intelectual e aprovação da versão final a ser publicada. 


\section{Referências}

1. Joint United Nations. Program on HIV/AIDS UNAIDS DATA 2018. Geneva: Joint United Nations Program on HIV/AIDS; 2018.

2. European Centre for Disease Prevention and Control, WHO Regional Office for Europe. HIV/AIDS surveillance in Europe 2018-2017 data. Copenhagen: WHO Regional Office for Europe; 2018.

3. Instituto Nacional de Saúde Dr. Ricardo Jorge. Infeção VIH e SIDA: a situação em Portugal a 31 de dezembro de 2017. Lisboa; Instituto Nacional de Saúde Dr. Ricardo Jorge; 2018.

4. Direção Geral da Saúde. Infeção VIH e SIDA - Desafios e Estratégias 2018. Lisboa: Direção Geral da Saúde; 2018.

5. Kim YK, Small E, Okumu M. School-based HIV/AIDS education, risky sexual behaviors, and HIV testing among high school students in the United States. Soc Work Health Care. 2019;58(3):258-73.

6. Cunha-Oliveira A Caramelo F, Patrício M, Camarneiro A, MassanoCardoso S, Pita J. Impacto de um programa de intervenção educativa nos comportamentos sexuais de jovens universitários. Rev Enf Referência. 2017;13(IV):71-82.

7. Mackman S, Hussein I. Awareness, knowledge, and behavior regarding HIV/AIDS among freshman students at Oakland University. EuroMediter Biomed J. 2017;12(16):73-6.

8. Bezerra E, Pereira M, Chaves A, Monteiro P. Representações sociais de adolescentes à cerca da relação sexual e do uso de preservativo. Rev Gaúcha Enferm. 2015;36(1):89-91.

9. Costa A, Araújo M, Araújo T, Gubert F, Vieira N. Protagonism of adolescents in preventing sexually transmitted diseases. Acta Paul Enferm. 2015;28(5):482-7.
10. Oliveira A. Preservativo, sida e saúde pública: factores que condicionam a adesão aos mecanismos de prevenção do VIH-SIDA. Coimbra: Universidade de Coimbra; 2008.

11. Cunha-Oliveira A, Cunha-Oliveira J, Cardoso I, Pita JR, MassanoCardoso S. Adaptação para a língua portuguesa da Escala de Embaraço Face ao Preservativo. Interações. 2011; 11(20):133-50.

12. Slaymaker E. A critique of international indicators of sexual risk behaviour. Sex Transm Infect. 2004;80 Suppl 2:ii13-ii21.

13. Cunha-Oliveira A, Cunha-Oliveira J, Massano-Cardoso S. VIH/ Sida: situação da prevenção em Portugal e o contexto europeu. Debater a Europa. 2016;14(14):141-74.

14. Direção Geral da Saúde. Programa nacional para a infeção VIH, Sida e tuberculose. Lisboa: Direção Geral da Saúde; 2017.

15. Joint United Nations Programme on HIV/AIDS (UNAIDS). 90-90-90. An ambitious treatment target to help end the AIDS epidemic. Geneva: UNAIDS; 2014

16. Morales A, Espada JP, Orgilés M. Barreras hacia la prueba de deteccíon del VIH en adolescentes en España. Psychosoc Intervent. 2016;25(3):135-41.

17. República Portuguesa. Decreto-lei $n^{0} 3 / 84$. Educação sexual e planeamento familiar. D.R. $1^{\text {a }}$ Série.71; 24 mar 1984. p. 981-3.

18. Tsala Dimbuene Z, Kuate Defo B. Fostering accurate HIV/AIDS knowledge among unmarried youths in Cameroon: do family environment and peers matter? BMC Public Health. 2011;11(1):348.

19. Reis M, Ramiro L, Matos M, Diniz J. Determinants influencing male condom use among university students in Portugal. Int J Sex Health. 2013;25(2):115-27.

20. Vasilenko SA, Kreager DA, Lefkowitz ES. Gender, Contraceptive Attitudes, and Condom Use in Adolescent Romantic Relationships: A Dyadic Approach. J Res Adolesc. 2015;25(1):51-62. 